\title{
On massive Nambu-Goldstone fields
}

\author{
Ivan Kharuk $^{1,2, *}$ and Andrey Shkerin ${ }^{3,2, * *}$ \\ ${ }^{1}$ Moscow Institute of Physics and Technology, Institutskii per. 9, Dolgoprudny 141700, Moscow Re- \\ gion, Russia, \\ ${ }^{2}$ Institute for Nuclear Research of the Russian Academy of Sciences, 60th October Anniversary \\ Prospect, 7a, 117312 Moscow, Russia, \\ ${ }^{3}$ Institute of Physics, Ecole Polytechnique Fédérale de Lausanne (EPFL), CH-1015, Lausanne, Switzer- \\ land
}

\begin{abstract}
We consider two theories undergoing the same spontaneous symmetry breaking pattern but with different representations of an order parameter under the Lorentz group. The effective description of the first theory includes a massive Nambu-Goldstone field, while in the second example it is absent. Based on this, we clarify the physical meaning of massive Nambu-Goldstone fields as non-radial modes needed to describe the fluctuations of an order parameter. The connection with the inverse Higgs phenomenon is also discussed.
\end{abstract}

\section{Introduction}

It is known that in the case of spontaneous breakdown of spacetime symmetries some of the Nambu-Goldstone (NG) fields ${ }^{1}$ can be redundant or massive [1-5]. Moreover, it is known that UV completion of such theories may be non-trivial due to the necessity of introducing massive non-radial modes [6]. In this letter, we demonstrate that these two observations are closely related to each other. For this purpose, we consider two theories undergoing the same spontaneous symmetry breaking (SSB) pattern but with order parameters belonging to different representations of the spacetime symmetry group. As a result, the effective description of the first theory includes massive Nambu-Goldstone ( $\mathrm{mNG}$ ) modes, while in the second example they are absent. Generalizing this observation, we conclude that $\mathrm{mNG}$ fields are present in an effective theory when they are needed to provide the full set of independent fluctuations of the vacuum. Otherwise, these fields are redundant and must be excluded from the theory by the means of the inverse Higgs phenomenon [1-3]. In particular, our results imply that the massive non-radial modes observed in [6] are nothing but $\mathrm{mNG}$ fields whose presence is necessary in order to UV complete the theory.

The letter is organized as follows. In section 2.1 we study an example of a theory whose effective description includes $\mathrm{mNG}$ fields. In section 2.2 another model is considered, which undergoes the same SSB pattern, but this time without $\mathrm{mNG}$ fields in the effective Lagrangian. Section 3 is dedicated to reconsidering the two examples within the coset space framework. We summarize the results and conclude the letter in section 4 .

\footnotetext{
*e-mail: ivan.kharuk@phystech.edu

**e-mail: andrey.shkerin@epfl.ch

${ }^{1}$ By Nambu-Goldstone fields we understand the degrees of freedom (DoF) associated with the action of the broken generators on the vacuum.
} 


\section{Two examples}

The theories we consider below are defined on $d$-dimensional Euclidean space. This allows us to disregard the question of stability of solutions and concentrate on their symmetry aspects.

\subsection{Physical mNG fields}

The symmetries of the first theory are $d$-dimensional internal and spacetime Poincare groups, $I S O(d)_{i n t}$ and $I S O(d)_{s t}$ accordingly. The theory contains two fields. The first one is a spin-0 field belonging to the vector representation of $S O(d)_{\text {int }}$ and on which internal translations act as shifts,

$$
\varphi^{a}(x) \rightarrow \Omega_{b}^{a} \varphi^{b}(x)+c^{a}, \quad \Omega_{b}^{a} \in S O(d)_{i n t}, \quad c^{a} \in \mathbb{R} .
$$

The second field, $V_{a}^{i}(x)$, belongs to a vector and co-vector representations of $S O(d)_{s t}$ and $S O(d)_{i n t}$ correspondingly, with the internal translations realized trivially,

$$
V_{a}^{i}(x) \rightarrow \Omega_{a}^{b} \Lambda_{j}^{i} V_{b}^{j}(x), \quad \Omega_{a}^{b} \in S O(d)_{i n t}, \quad \Lambda_{j}^{i} \in S O(d)_{s t} .
$$

The Lagrangian of the theory reads

$$
\mathcal{L}=-\frac{1}{2}\left(\partial_{i} \varphi^{a}\right)^{2}+\frac{1}{4}\left(\partial_{[i} V_{j]}^{a}\right)^{2}+\varkappa V_{a}^{i} \partial_{i} \varphi^{a}+\frac{\lambda}{4 d}\left(V_{a}^{i} V_{i}^{a}-d M_{V}^{2}\right)^{2}
$$

where $\varkappa, \lambda$, and $M_{V}$ are some positive constants and the square brackets stay for antisymmetrization in the corresponding indices. The potential for $V_{a}^{i}$ is chosen so that $V_{a}^{i}$ develops a non-zero vacuum expectation value (vev). Because of the interaction between the fields, $\varphi^{a}$ is forced to have a non-zero vev as well. Assuming $\lambda M_{V}^{2}>\varkappa^{2}$, the vacuum solution reads

$$
\varphi^{a}=\mu^{2} x^{a}, \quad V_{a}^{i}=M \delta_{a}^{i}, \quad M=\sqrt{M_{V}^{2}-\frac{\varkappa^{2}}{\lambda}}, \mu^{2}=\varkappa M
$$

The SSB pattern corresponding to this solution is

$$
I S O(d)_{s t} \times I S O(d)_{i n t} \rightarrow I S O(d)_{V},
$$

where $I S O(d)_{V}$ is a semidirect product of $P_{V}^{i}=P_{s t}^{i}-\mu^{2} P_{i n t}^{i}$ and $S O(d)_{V}$, a diagonal subgroup of $S O(d)_{s t} \times S O(d)_{i n t}$. Since only $S O(d)_{V}$ is left unbroken, in what follows we do not distinguish between the spatial and internal indices. The NG fields are then given by the translations of $\varphi^{a}$ and simultaneous internal rotations of $\varphi^{a}$ and $V_{a}^{i}$. To simplify the calculations and ensure that the coordinates do not enter the effective Lagrangian, we parametrize the fluctuations of the vacuum as follows,

$$
\varphi^{a}(x)=\mu^{2} x^{a}+\psi^{a}(x), \quad V_{a}^{i}(x)=\Omega_{a}^{i}(x) M, \quad \Omega_{a}^{i}=\delta_{a}^{i}+\omega_{a}^{i}-\frac{1}{2} \omega_{b}^{i} \omega_{a}^{b}+\ldots,
$$

where dots stay for higher order terms in $\omega_{a}^{i}$. Substituting this expression back into eq. (3), one obtains the effective Lagrangian of the theory, which to the leading order in $\omega_{a}^{i}$ reads

$$
\mathcal{L}_{\psi, A}=-\frac{1}{2}\left(\partial_{i} \psi^{a}\right)^{2}+\frac{1}{4}\left(\partial_{[i} A_{j]}^{a}\right)^{2}-\frac{1}{2} \varkappa^{2} A_{j}^{i} A_{i}^{j}+\varkappa A_{a}^{i} \partial_{i} \psi^{a},
$$

where we have switched to the canonically normalized filed $A_{a}^{i}=M \omega_{a}^{i}$. To diagonalize the Lagrangian, we redefine the DoF as follows,

$$
\varkappa A_{i j}=\partial_{i} \psi_{j}-\partial_{j} \psi_{i}+\varkappa \tilde{A}_{i j} .
$$


We will discuss this field redefinition in the context of inverse Higgs phenomenon in the next section. In the new variables, the Lagrangian becomes

$$
\mathcal{L}_{\psi, \tilde{A}}=-\frac{1}{4}\left(\left(\partial_{i} \psi^{a}\right)^{2}+\left(\partial_{a} \psi^{a}\right)^{2}\right)-\frac{\varkappa^{2}}{2} \tilde{A}_{j}^{i} \tilde{A}_{i}^{j}+\frac{1}{4}\left(\partial_{[i} \tilde{A}_{j]}^{k}\right)^{2} .
$$

An important observation is that besides $\psi^{a}$, this Lagrangian also contains the massive antisymmetric field $\tilde{A}_{a}^{i}$, which is not a radial mode. Note also that $\tilde{A}_{a}^{i}$,s mass depends on $\varkappa$, not $\mu$, and, hence, is not fixed by the SSB pattern alone.

Let us discuss the physical nature of the field $\tilde{A}_{j}^{i}$. As it can be verified, $\tilde{A}_{j}^{i}$ transforms linearly under the action of all symmetry generators. In this sense, it does not represent a conventional NG field. However, since $\widetilde{A}_{j}^{i}$ is associated with the fluctuations of the vacuum (and, hence, must be present in the effective theory), we believe that it is physically justified to consider it on equal grounds with the NG fields. Note also that $\tilde{A}_{j}^{i}$ can be distinguished from ordinary matter fields. Indeed, the structure of a kinetic term of an ordinary matter field is only restricted by the general requirement of invariance under the symmetry group. The full kinetic term for $\tilde{A}_{j}^{i}$, on the other hand, is restricted to have the form

$$
\mathcal{L}_{\tilde{A}, k i n .}=\frac{1}{4}\left(\partial_{[i} \Omega_{j]}^{a}\left(\tilde{A}_{l}^{k} / M\right)\right)^{2} .
$$

Going back to the discussion of the effective Lagrangian, we notice that at the energy scales below $\varkappa$ the field $\tilde{A}_{a}^{i}$ can be integrated out. After doing so, one obtains the low-energy Lagrangian

$$
\mathcal{L}_{\psi}=-\frac{1}{4}\left(\left(\partial_{i} \psi^{a}\right)^{2}+\left(\partial_{a} \psi^{a}\right)^{2}\right)
$$

describing the massless mode $\psi^{a}$.

To summarize, we considered the example of a theory whose effective description involves an $\mathrm{mNG}$ field. At the energies below the strong-coupling scale $M$ the theory is described by the Lagrangian (7) containing the gapped and gapless modes. The dynamics of the theory in the low-energy limit is governed by the Lagrangian (11) containing the gapless NG field only.

\subsection{Redundant mNG fields}

Now we would like to provide an example of a theory undergoing the same SSB pattern as in the previous case but without $\mathrm{mNG}$ fields. For this purpose, we introduce a new scalar field $\theta$ and take the Lagrangian in the form

$$
\mathcal{L}=-\frac{1}{2}\left(\square \varphi^{a}\right)^{2}-\frac{1}{2}\left(\partial_{i} \theta\right)^{2}+\frac{1}{4}\left(\partial_{[i} V_{j]}^{a}\right)^{2}+\lambda \theta V_{a}^{i} \partial_{i} \varphi^{a},
$$

where $\square=\partial_{i} \partial^{i}$ and $\lambda$ is a constant. This theory has the same symmetries as the previous example but the presence of $\theta$ and the box operator ensure that

$$
\varphi^{a}=\mu^{2} x^{a}, \quad \theta=0, \quad V_{a}^{i}=0
$$

is a solution of the equation of motion with an arbitrary $\mu^{2}$. Background (13) invokes the same SSB pattern as before, eq. (5). The crucial observation is that now one can reproduce all possible fluctuations of the vacuum configuration by acting on it by the generators of the broken internal translations only. As a consequence, the NG fields for the broken Lorentz transformations are redundant [2-4]. To put it in other words, the NG sector of the effective theory contains just $d$ DoF describing the fluctuations of $\varphi^{a}$. 
Straightforward calculations yield the effective Lagrangian of the theory to be

$$
\mathcal{L}_{\psi}=-\frac{1}{2}\left(\square \psi^{a}\right)^{2}-\frac{1}{2}\left(\partial_{i} \theta\right)^{2}+\frac{1}{4}\left(\partial_{[i} V_{j]}^{a}\right)^{2}+\lambda \theta V_{a}^{i}\left(\mu^{2} \delta_{i}^{a}+\partial_{i} \psi^{a}\right),
$$

where $\psi^{a}$ is the fluctuation of $\varphi^{a}$ on top of background (13). We note that the only field undergoing SSB is $\varphi^{a}$, while $V_{a}^{i}$ and $\theta$ are spectators and should be considered as matter fields in the low-energy phase. An important point is that $V_{a}^{i}$ is charged under the action of $S O(d)_{\text {int }}$, while a low-energy observer would introduce fields as linear representations of $S O(d)_{V}$. To rewrite Lagrangian (14) in the appropriate from the low-energy perspective form, note that the field $V_{a}^{i}$ can be redefined as follows,

$$
V_{a}^{i} \rightarrow \Omega_{a}^{b}\left(\psi^{c}\right) \tilde{V}_{b}^{i}, \quad \Omega_{i j}\left(\psi^{c}\right)=e^{i\left(\partial_{i} \psi_{j}-\partial_{j} \psi_{i}\right)},
$$

where $\Omega_{a}^{b}$ belongs to $S O(d)_{\text {int }}$. This allows to consider the transformation of $V_{a}^{i}$ under the action of $S O(d)_{i n t}$ as the transformation of $\Omega_{a}^{b}$ which leaves $\tilde{V}_{a}^{i}$ unchanged. Moreover, since the action of $S O(d)_{s t}$ can always be completed to the action of $S O(d)_{V}$ and $S O(d)_{\text {int }}$, this allows to uncharge $V_{a}^{i}$ under the action of $S O(d)_{S T}$ as well. Substituting eq. (15) into Lagrangian (14), one arrives at the valid low-energy description of the theory.

\section{A view from coset space approach}

In this section we reconsider the examples above from the perspective of the coset space technique. Following the standard rules [7, 8] for SSB pattern (5), we take the coset space

$$
g_{H}=e^{i P_{V i} x^{i}} e^{i \bar{P}_{a} \psi^{\prime a}} e^{\frac{i}{2} \bar{M}_{a b} \omega^{\prime a b}},
$$

where $\psi^{\prime a}$ and $\omega^{\prime a b}$ are the NG fields for the broken internal translations and rotations correspondingly and the unbroken combination of translations ${ }^{2}$ give rise to the coordinates in the broken phase of the theory. First, we would like to find the transformation laws for the NG fields introduced in this way. For this purpose, we act by $e^{i \bar{P}_{a} q^{a}}$ and $e^{i \bar{M}_{a b} \alpha^{a b}}$ on coset space (16) with constant parameters $q^{a}$ and $\alpha^{a b}$. The transformation laws of $\psi^{\prime a}$ and $\omega^{\prime a b}$ are found to be

$$
\begin{gathered}
e^{i \bar{P}_{a} q^{a}}: \quad \psi^{\prime a} \rightarrow \psi^{\prime a}+q^{a}, \omega^{\prime a b} \rightarrow \omega^{\prime a b}, \\
e^{i \bar{M}_{a b} \alpha^{a b}}: \quad \psi^{\prime a} \rightarrow \Omega(\alpha)_{b}^{a} \psi^{\prime b}+\left(\Omega(\alpha)_{b}^{a}-\delta_{b}^{a}\right) x^{b}, \quad \omega^{\prime a b} \rightarrow \omega^{\prime a b}+\alpha^{a b}+\ldots,
\end{gathered}
$$

where dots stay for higher order terms. Further, to obtain the ingredients for the construction of the effective theory, we find the Maurer-Cartan forms for coset space (16),

$$
g_{H} d g_{H}^{-1}=i \omega_{P_{V}}^{i} P_{V i}+i \omega_{\bar{P}}^{a} \bar{P}_{a}+i \omega_{\bar{M}}^{a b} \bar{M}_{a b} .
$$

To the leading order they are given by

$$
\omega_{P_{V}}^{i}=d x^{i}, \quad \omega_{\bar{P}}^{a}=d \psi^{\prime a}-\mu^{2} \omega_{b}^{\prime a} d x^{b}, \quad \omega_{\bar{M}}^{\mu a}=d \omega^{\prime \mu a} .
$$

From eq. (19) one finds the tetrads, the metric, and the covariant derivatives of the NG fields,

$$
e_{j}^{i}=\delta_{j}^{i}, \quad g_{i j}=e_{i}^{k} e_{j}^{l} \delta_{k l}=\delta_{i j}, \quad D_{i} \psi^{\prime a}=\partial_{i} \psi^{\prime a}-\mu^{2} \omega_{i}^{\prime a}, \quad D_{i} \omega^{\prime a b}=\partial_{i} \omega^{\prime a b} .
$$

\footnotetext{
${ }^{2}$ We call a generator unbroken if its action on the vacuum is trivial. This allows us to distinguish between non-linearly realized generators and broken ones.
} 
First, we would like to note that by using the ingredients above one can reproduce the effective Lagrangian of section 2.1, the one with the massive Goldstone field. Indeed, as it can be verified, $\psi^{a}$ and $\omega_{a}^{i}$ defined in eq. (6) have the same transformation properties as the NG fields defined in eq. (16), and, hence, represent the same DoF. Further, the part of the effective Lagrangian containing the kinetic term for $\psi^{\prime a}$, the mass term for $\omega_{a}^{\prime i}$, and their interaction, can be reproduced in the coset space framework as

$$
-\frac{1}{2}\left(D_{i} \psi^{\prime a}\right)^{2}=-\frac{1}{2}\left(\partial_{i} \psi^{\prime a}\right)^{2}-\frac{1}{2} \varkappa^{2} A_{a}^{i} A_{a}^{i}+\varkappa A_{a}^{i} \partial_{i} \psi^{\prime a},
$$

where we have switched to the canonically normalized field $A_{a}^{i}=M \omega_{a}^{i}$. Upon identification $\psi^{\prime a}=\psi^{a}, \omega^{\prime a b}=\omega^{a b}$, this coincides with the corresponding part of eq. (7). $A_{a}^{i}$, s kinetic term can be reproduced straightforwardly, since the covariant derivative of $\omega^{a b}$ coincides with the usual partial derivative. We conclude that Lagrangian (7) can be fully reproduced within the coset space approach.

Let us now make contact with the inverse Higgs phenomenon. Since it is considered as a way of reducing the number of NG fields in the effective theory, we expect that the effective Lagrangian of section 2.2 can be reproduced in this way. inverse Higgs constraints for coset space (16) are

$$
D_{[i} \psi_{j]}=0: \quad \partial_{i} \psi_{a}-\partial_{a} \psi_{i}=\varkappa A_{i a} .
$$

We know that the field $A_{a}^{i}$ is unphysical in this case, and, hence, must be eliminated in the coset space framework. This suggests the following interpretation of eq. (22): if $A_{a}^{i}$ were physical, then they would have the same transformation properties as the antisymmetric derivative of $\psi^{a}$. Taking this into account, one can parametrize the coset space for the theory of section 2.2 as follows,

$$
g_{H}=e^{i P_{V i} x^{i}} e^{i \bar{P}_{a} \psi^{\prime a}} e^{\frac{i}{2} \bar{M}_{a b}\left(\partial^{a} \psi^{\prime b}-\partial^{b} \psi^{\prime a}\right)} .
$$

Such parametrization of the coset automatically ensures that there are only $d$ NG modes. Moreover, a closer inspection of this expression from the perspective of reducing matrices $[9,10]$, also known as the polar decomposition, shows that this automatically uncharges all of the fields under the action of $S O(d)_{i n t}$ and $S O(d)_{s t}$. Hence, matter fields are introduced as representations of $S O(d)_{V}$, as they should be. Thus, in the theory of section 2.2 the $\mathrm{mNG}$ field $A_{a}^{i}$ should be considered as an auxiliary field which is to be excluded from the theory via the imposition of the inverse Higgs constraints. From this point of view, the inverse Higgs phenomenon is a tool that allows to uncharge the fields under the action of all symmetries except $S O(d)_{V}$. As it can be verified, this indeed allows to reproduce the effective Lagrangian for the theory (12).

Let us now discuss the role of the inverse Higgs phenomenon in the theory of section 2.1 , which includes the physical $\mathrm{mNG}$ field. Because of the symmetry restrictions, in the absence of matter fields the inverse Higgs constraints give the expression for $A_{j}^{i}$ in terms of $\psi^{a}$ one would have obtained by integrating $A_{j}^{i}$ out $[3,11]$. Hence, by using the left covariant derivatives, one can reproduce the low-energy limit of the theory,

$$
\mathcal{L}_{\psi}=-\frac{1}{8}\left(D_{\{i} \psi_{j\}}\right)^{2}
$$

which coincides with (11).

Note, however, that, instead of imposing the inverse Higgs constraints, one can introduce a new field $\tilde{A}_{j}^{i}$ via

$$
D_{[i} \psi_{j]}=\tilde{A}_{j}^{i}
$$


Since the 1.h.s. of eq. (25) contains $A_{j}^{i}$ without derivatives, it represents a valid change of variables. Importantly, it allows us to switch from the field $A_{j}^{i}$, which transforms nonhomogeneously under the action of the symmetry group, to the field $\tilde{A}_{j}^{i}$ transforming linearly under the full symmetry group. The field $\tilde{A}_{j}^{i}$ is an analog of massive non-radial modes observed in [6]. As we see, this field transform linearly under the action of the broken generators and must be present in a theory in order to complete the set of possible fluctuations of a vacuum. The DoF redefinition (25) is precisely the change of variable we made before, eq. (8), and corresponds to extracting the "the inverse Higgs" part of the field.

\section{Conclusion}

From the analysis above we conclude that the question of whether to impose the inverse Higgs constraints or not is, in fact, misleading. These constraints can always be imposed, since, by themselves, they do not reduce the amount of NG fields. The physically meaningful question to ask is whether an effective description of a theory contains massive non-radial modes. As we demonstrated, the answer depends on the representation of an order parameter: if such modes are needed to describe the full set of fluctuations of the vacuum, they will be present in the effective theory, and vice versa. We believe that this result clarifies the connection between the mNG fields and the inverse Higgs phenomenon.

\section{Acknowledgments}

The authors thank E. Ivanov, R. Penco and S. Sibiryakov for useful discussion. The work of I.K. was supported by the Grant 14-22-00161 of the Russian Science Foundation. The work of A.S. was supported by ERC-AdG-2015 grant 694896.

\section{References}

[1] E.A. Ivanov, V.I. Ogievetsky, Teor. Mat. Fiz. 25, 164 (1975)

[2] I. Low, A.V. Manohar, Phys. Rev. Lett. 88, 101602 (2002), hep-th/0110285

[3] A. Nicolis, R. Penco, F. Piazza, R.A. Rosen, JHEP 11, 055 (2013), 1306.1240

[4] H. Watanabe, H. Murayama, Phys. Rev. Lett. 110, 181601 (2013), 1302 . 4800

[5] T. Brauner, H. Watanabe, Phys. Rev. D89, 085004 (2014), 1401.5596

[6] S. Endlich, A. Nicolis, R. Penco, Phys. Rev. D89, 065006 (2014), 1311.6491

[7] V. Ogievetsky, Nonlinear realization of internal and space-time symmetries, in X-th winter school of theoretical physics in Karpacz, Poland (1974)

[8] S.R. Coleman, J. Wess, B. Zumino, Phys. Rev. 177, 2239 (1969)

[9] A. Salam, J.A. Strathdee, Phys. Rev. 184, 1750 (1969)

[10] S. Weinberg, The quantum theory of fields. Vol. 2: Modern applications (Cambridge University Press, 2013), ISBN 9781139632478, 9780521670548, 9780521550024

[11] L.V. Delacrétaz, S. Endlich, A. Monin, R. Penco, F. Riva, Journal of High Energy Physics 2014, 8 (2014) 